\title{
Effects of Intermodulation Distortion on the Performance of a Hybrid Radio/Fiber System Employing a Self-Pulsating Laser Diode Transmitter
}

\author{
A. Kaszubowska, L. P. Barry, Member, IEEE, and P. Anandarajah
}

\begin{abstract}
A self-pulsating laser is used to generate a multicarrier (five radio frequency (RF) channels) microwave optical signal for use in a hybrid radio/fiber system. The self-pulsation is achieved by external light injection into the laser diode. By varying the RF channel spacing, we have been able to estimate the degradation in system performance due to intermodulation distortion (caused by the nonlinear dynamic response of the laser). The power penalty on the central RF channel is found to be $3.2 \mathrm{~dB}$ for operation at the RF band around the laser self-pulsation frequency of $18.5 \mathrm{GHz}$. We have also characterized the performance of the multicarrier hybrid radio/fiber system in the frequency band corresponding to the inherent relaxation frequency of the laser.
\end{abstract}

Index Terms-External light injection, microwave photonics, multicarrier system, optical communications, optical systems, self-pulsation, semiconductor laser diode.

\section{INTRODUCTION}

$\mathbf{H}$ IGH-CAPACITY mobile networks of the future will probably use high-frequency microwave signals as the access medium (15-60 GHz), as this offers a large bandwidth for data transfer. These systems are likely to employ an architecture in which signals are generated at a central location and then distributed to remote base stations over optical fiber, before being transmitted over small areas using microwave antennas [1], [2]. This type of architecture should prove to be highly cost efficient since it allows sharing of the transmission and processing equipment between many base stations. It is also expected that these broad-band networks will divide the available bandwidth into a number of radio frequency (RF) channels for broadcasting data "over the air." This use of multiple carrier distribution is required in high-capacity multipath environments to overcome multipath fading effects.

On the transmission side of a radio over fiber distribution network, it is necessary to generate the microwave optical data signal using semiconductor laser diodes. The simplest technique available involves direct modulation of the laser with the microwave data carriers. However, the limited bandwidth of laser diodes means that we are normally unable to use high-frequency $\mathrm{RF}$ carriers $(>10 \mathrm{GHz})$. We have recently demonstrated the use of external injection to greatly improve the frequency response of a laser at RF bands well beyond the inherent relaxation frequency of the laser, and we have shown how this technique may

Manuscript received December 16, 2002; revised February 12, 2003.

The authors are with the School of Electronic Engineering, Dublin City University, Dublin 9, Ireland (e-mail: liam.barry@dcu.ie).

Digital Object Identifier 10.1109/LPT.2003.811330 be used for greatly improving the performance of a single RF channel, and a dual RF channel, hybrid radio/fiber system [3], [4]. The enhancement in system performance is a result of the laser diode self-pulsating as we externally inject light into the diode, and we have completely characterized this self-pulsation in [4]. As we only employed two RF data channels in this previous work, we were unable to ascertain how the nonlinear dynamic response of the laser would affect the system performance via intermodulation distortion (IMD) in the multicarrier system [5]-[7]. In this letter, we have thus used the externally injected self-pulsating laser for the generation of a multicarrier microwave photonic signal with five RF data channels. By varying the spacing between these RF channels we have been able to determine how the dynamic nonlinearity of the self-pulsating laser affects the system performance via IMD, and we show a 3.2-dB power penalty for the central RF channel due to IMD. We have also examined the system performance of the hybrid/radio fiber setup when using the free-running laser (without external injection) at RF bands corresponding to 1) the laser's relaxation oscillation frequency, and 2) the linear section of the laser's modulation response. This allows us to compare and contrast how the different dynamic responses of the laser affect the performance of our microwave photonic system.

\section{MulticARrier RAdio-Over-Fiber SetuP}

The laser diode used for the experiments is a standard multiple quantum-well distributed-feedback (DFB) device from NEL. The laser has a threshold current of $26 \mathrm{~mA}$, and an intrinsic modulation bandwidth of around $8 \mathrm{GHz}$. By injecting light from a wavelength tunable external cavity laser, around the same wavelength as the DFB emission wavelength, we significantly alter the modulation response of the device and can achieve excellent response at frequencies from 14 to $25 \mathrm{GHz}$, due to the laser beginning to self-pulsate. The self-pulsation frequency is determined by the power level of the externally injected signal [4]. Fig. 1 displays the modulation response of the laser with and without external injection, (injection level of $0 \mathrm{dBm}$ and laser biased at $60 \mathrm{~mA}$, in both cases).

To investigate the use of the externally injected self-pulsating laser as the transmitter for a multicarrier radio-over-fiber distribution system, we have employed the experimental setup shown in Fig. 2. We initially couple together a $300-$ and a $600-\mathrm{MHz}$ local oscillator signal and then mix the combined signal with a $155-\mathrm{Mb} / \mathrm{s}$ nonreturn-to-zero data stream from an Anritsu pattern generator that has been passed through a 117-MHz low-pass 


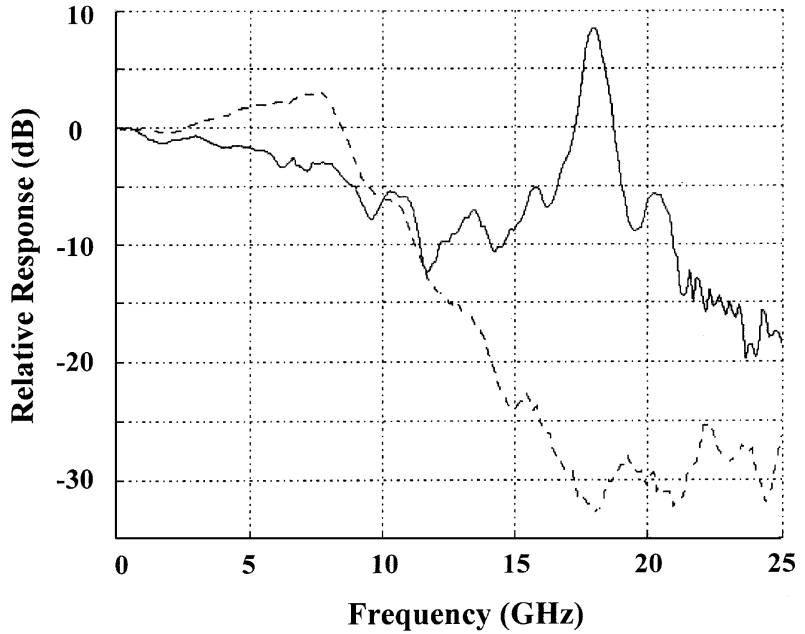

Fig. 1. Modulation response of free-running laser (dashed line), and laser with external injection level of $0 \mathrm{dBm}$ (solid line) resulting in $18.5-\mathrm{GHz}$ self-pulsation. Laser biased at $60 \mathrm{~mA}$ in both cases.

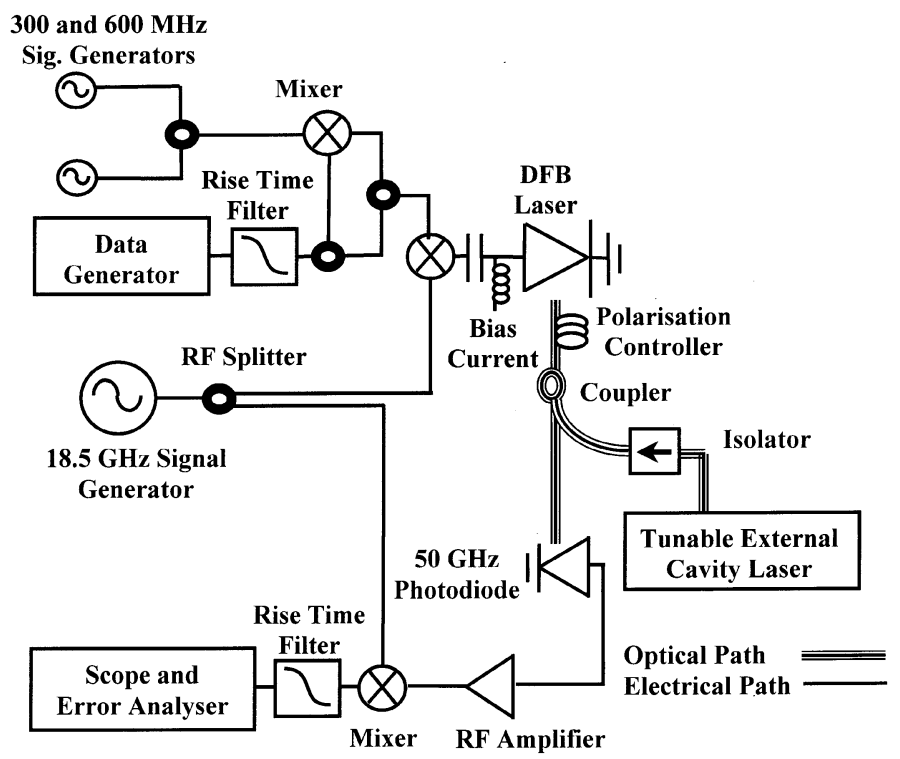

Fig. 2. Experimental setup for five-channel multicarrier hybrid radio/fiber system using self-pulsating laser diode.

filter (to minimize the bandwidth of the electrical data signal). The resulting signal is then coupled together with another part of the $155-\mathrm{Mb} / \mathrm{s}$ data signal from the pattern generator, and the overall signal is then mixed with a $18.5-\mathrm{GHz}$ carrier. This leads to the generation of five binary phase-shift keyed RF data channels centered at $18.5 \mathrm{GHz}$ with $300-\mathrm{MHz}$ spacing between the channels. It should be noted that the two base-band data signals from the pattern generator are propagated through cables with a length difference of $3 \mathrm{~m}$ before being mixed with the appropriate local oscillators. This ensures minimum correlation between the central RF channel (test channel), and the higher and lower frequency channels (interfering channels), as $3 \mathrm{~m}$ corresponds to around 27 ns delay which is more than four bit slots for the $155-\mathrm{Mb} / \mathrm{s}$ data stream.

The five-channel RF data signal is then used to directly modulate the DFB laser diode described above. The laser is biased at $60 \mathrm{~mA}$, and has an external injection level of $0 \mathrm{dBm}$ from a

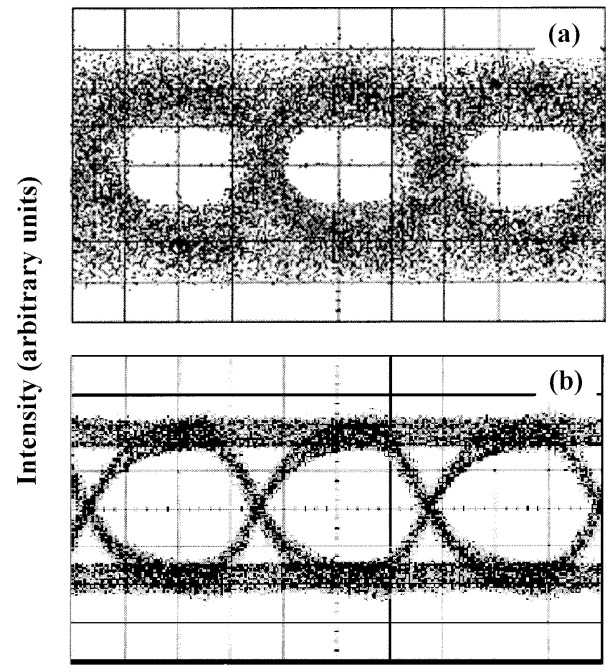

Time (2 ns/div)

Fig. 3. Received eye diagram of the recovered $155-\mathrm{Mb} / \mathrm{s}$ data signal at the central RF carrier frequency when (a) the RF carriers are equally spaced (maximum IMD), and (b) the RF carriers are unequally spaced. Received optical power (before photodiode) was $-8 \mathrm{dBm}$ in both cases.

wavelength tunable source to facilitate the self-pulsation around the 18.5-GHz RF band. The resulting optical microwave data signal from the laser is then detected with a $50-\mathrm{GHz}$ pin photodiode. In a complete radio/fiber system, the detector output would be transmitted through an RF antenna to the mobile stations, however, in our experiment we have concentrated on the optical part of the system, hence the down conversion of the $\mathrm{RF}$ carrier data signals takes place after the photodiode. To recover the central RF data channel, we have down-converted it by mixing the detected signal with the $18.5-\mathrm{GHz}$ local oscillator. The down-converted signal (central channel of the five-channel RF data signal) is then passed through a low-pass filter to ensure that only the required base-band signal is examined using the 50-GHz oscilloscope and error analyzer.

Fig. 3(a) displays the received eye diagram of the down-converted $155-\mathrm{Mb} / \mathrm{s}$ data signal after propagation through the optical microwave link when the received optical power level is $-8 \mathrm{dBm}$. By varying the detected optical power level using an optical attenuator, and measuring the bit-error rate (BER) of the received signals, we can plot system performance against received optical power (Fig. 4). We then proceeded to vary the frequency spacing of the five-channel RF system. The aim of this is to determine how the IMD due to mixing between Channels 1 and 2, and Channels 4 and 5, affects the performance of the central channel. Fig. 3(b) displays the received eye diagram for a received optical power of $-8 \mathrm{dBm}$, and Fig. 4 shows the BER versus received optical power, when the frequencies of Channels 1 and 5 are offset by a further $125 \mathrm{MHz}$ from the central $\mathrm{RF}$ channel. The result of this is to ensure the mixing products of Channels 1 and 2, and Channels 4 and 5, are no longer at the same frequency as the central data channel. We can clearly see that in this case the system performance is improved by $3.2 \mathrm{~dB}$ for a received BER of $10^{-7}$, and the improvement in the received eye diagram for the same detected optical power level is very noticeable. The degradation in system performance, when 


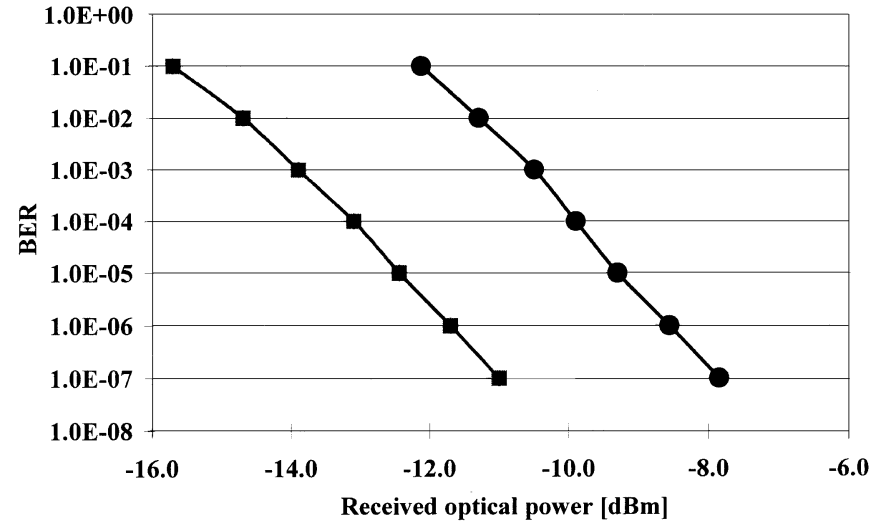

Fig. 4. BER versus received optical power for the central RF data channels using directly modulated self-pulsating laser for cases when (a) the RF carriers are equally spaced (circles) and (b) the RF carriers are unequally spaced (squares).

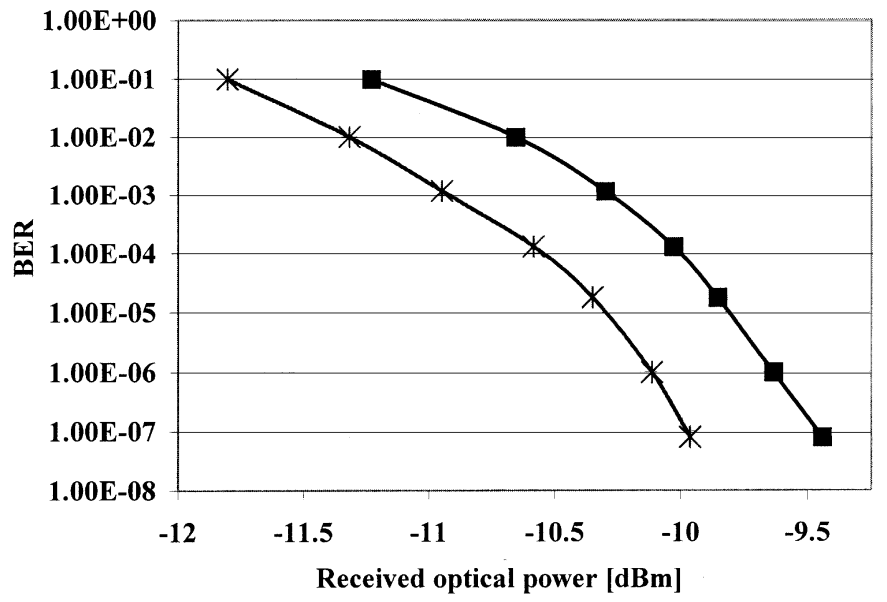

Fig. 5. BER versus received optical power for the central RF data channel using the RF band around the relaxation frequency of the free-running laser (no external injection). (a) RF carriers equally spaced (squares) and (b) RF carriers unequally spaced (crosses).

the RF channels are equally spaced, is a result of the IMD due to the dynamic nonlinearity of the self-pulsating laser.

We then carried out a similar experiment but changed the central frequency of the multicarrier RF data signal to 8.5 and $2 \mathrm{GHz}$ (with external injection into the DFB laser turned off such that it had its inherent modulation response, as displayed in Fig. 1). As we can see from this response, the relaxation frequency of the laser is around the RF band at $8.5 \mathrm{GHz}$. As before, we characterized the system performance of the central RF data channel when the RF channel spacing was equal (such that the IMD on the central channel is maximized), and when the spacing between Channels 1 and 2, and Channels 4 and 5, is increased by $125 \mathrm{MHz}$ such that the IMD on the central channel is minimized. Fig. 5 displays the system performance for both cases and we can see that the power penalty at a BER of $10^{-7}$ is only $0.6 \mathrm{~dB}$ when using the RF band around $8.5 \mathrm{GHz}$. When using the linear portion of the laser modulation response around $2 \mathrm{GHz}$, the penalty due to IMD was only $0.2 \mathrm{~dB}$.

\section{DiscuSSION AND CONCLUSION}

We have investigated the use of a self-pulsating laser diode (achieved using external injection into a commercial DFB laser) for the distribution of multicarrier RF data signals in a hybrid radio/fiber communication system. By altering the frequency spacing between the RF channels we have been able to ascertain the performance degradation in the hybrid radio/fiber system due to the nonlinearity of the modulation response around the self-pulsation frequency of the laser. The power penalty on the central channel of the five-channel data signal is found to be around $3.2 \mathrm{~dB}$ due to IMD. The significant power penalty is a result of the large dynamic nonlinearity around the laser's self-pulsation frequency. The result would suggest that for a multicarrier RF distribution system using a radio-over-fiber architecture with a self-pulsating laser as the transmitter, it may be necessary to employ unequal channel spacing to mitigate against IMD effects [8]. We have also examined the performance of our hybrid radio/fiber system when using the RF bands around the laser's relaxation frequency, and linear section of its modulation response, and found that in these cases the degradations in performance due to IMD are 0.6 and $0.2 \mathrm{~dB}$, respectively, for the central channel of the five-channel system. This result allows us to contrast how the dynamic nonlinearities due to the self-pulsation, and the relaxation oscillation, effect the performance of a multicarrier radio-over-fiber system.

\section{REFERENCES}

[1] R. P. Braun, G. Grosskopf, H. Heidrich, C. von Helmolt, R. Kaiser, K. Kruger, U. Kruger, D. Rohde, F. Schmidt, R. Stenzel, and D. Trommer, "Optical microwave generation and transmission experiments in the 12- and 60-GHz region for wireless communications," IEEE Trans. Microwave Theory Tech., vol. 46, pp. 320-330, Apr. 1998.

[2] Z. Ahmed, D. Novak, R. B. Waterhouse, and H.-F. Liu, "37-GHz fiberwireless system for distribution of broad-band signals," IEEE Trans. Microwave Theory Tech., vol. 45, pp. 1431-1435, Aug. 1997.

[3] A. Kaszubowska, P. Anandarajah, and L. P. Barry, "Improved performance of a hybrid radio/fiber system using a directly modulated laser transmitter with external injection," IEEE Photon. Technol. Lett., vol. 14, pp. 233-235, Feb. 2002.

[4] — " Multiple RF carrier distribution in a hybrid radio/fiber system employing a self-pulsating laser diode transmitter," IEEE Photon. Technol. Lett., vol. 14, pp. 1599-1601, Nov. 2002.

[5] J. Le Bihan and G. Yabre, "FM and IM intermodulation distortions in directly modulated single-mode semiconductor lasers," IEEE J. Quantum Electron., vol. 30, pp. 899-904, Apr. 1994.

[6] R. Hui, B. Zhu, R. Huang, C. T. Allen, K. R. Demarest, and D. Richards, "Subcarrier multiplexing for high-speed optical transmission," J. Lightwave Technol., vol. 20, pp. 417-427, Mar. 2002.

[7] H. M. Salgado and J. J. O'Reilly, "Accurate performance modeling of subcarrier multiplexed fiber/radio systems: Implications of laser nonlinear distortion and wide dynamic range," IEEE Trans. Commun., vol. 44, pp. 988-996, Aug. 1996.

[8] M. D. Atkinson, N. Santoro, and J. Urrutia, "Integer sets with distinct sums and differences and carrier frequency assignments for nonlinear repeaters," IEEE Trans. Commun., vol. 44, pp. 614-617, June 1986. 